\title{
On the Relations Between 2D and 3D Fractal Dimensions: Theoretical Approach and Clinical Application in Bone Imaging
}

\author{
H. Akkari ${ }^{a}$, I. Bhouri ${ }^{b}{ }^{1}$ P. Dubois ${ }^{c}$ and M.H. Bedoui ${ }^{a}$ \\ ${ }^{a}$ Laboratoire de Biophysique, TIM, Faculté de Médecine de Monastir, Tunisia \\ ${ }^{b}$ Unité de recherche Multifractals et Ondelettes, Faculté des Sciences de Monastir, Tunisia \\ ${ }^{b}$ INSERM, U 703, ITM, CHRU Lille, France
}

\begin{abstract}
The inner knowledge of volumes from images is an ancient problem. This question becomes complicated when it concerns quantization, as the case of any measurement and in particular the calculation of fractal dimensions. Trabecular bone tissues have, like many natural elements, an architecture which shows a fractal aspect. Many studies have already been developed according to this approach. The question which arises however is to know to which extent it is possible to get an exact determination of the fractal dimension of a given volume only from the fractal measurement made on the projections or slice images given by medical imaging. This paper gives general results about the Minkowski dimensions and contents of projections and sections of a set. We also show with examples that they depend essentially on the directions of the planes and so there is - in general case - no relation between 3D and 2D fractal dimensions. This consideration is then illustrated with examples from synthetic models and from CT scan images of wrists. In conclusion, this study reveals that the quantitative characterization of an organic volume (in particular osseous) requires taking into account the whole volume, and not only some of its slices or projections.
\end{abstract}

Key words: Minkowski dimensions and contents, Hausdorff measure, projection, sections, osteoporosis, medical imaging.

AMS subject classification: 28A78, 28A80, 28A75

\footnotetext{
${ }^{1}$ Corresponding author. E-mail: bhouri_imen@yahoo.fr
} 


\section{Introduction}

Osteoporosis is characterized by low bone mineral density (BMD) and micro architectural deterioration of bone tissue [6]. In clinical practice, the diagnosis of osteoporosis is mainly based on the measurement of bone mineral density. However, the risk of bone fragility results also from the changes in the bone architecture that is independent of bone density [20].

During the last decade, many researches have been directed toward the analysis of the trabecular micro architecture images, through either a morphological or a mathematical approach (e.g., statistical or fractal analysis) [10,11]. Other works have searched for correlations between the 3D characteristics of bone micro architecture (morphological, topological, or otherwise) and the parameters of $2 \mathbf{D}$ bone projections [9, 18].

Several methods such as fractal analysis have also been used to describe and analyze complex shapes and structural patterns. Fractal analysis expresses numerical values (Fractal dimensions) especially the Minkowski or box dimension which is one of the most widely used fractional dimensions: its popularity is largely due to its relative ease of rigorous computation and numerical estimation. This dimension has been used to evaluate the degradation of bone micro architecture and distinguishes between patients with and without osteoporosis. Several techniques have been used for the measurement of fractal dimension such as box counting method, method of Minkowski Bouligand and maximum likelihood method based on fractional Brownian motion $[3,10]$.

Trabecular bone is a complex 3D network of connected trabeculae. Consequently, 2D analysis is limited because it allows only the study of a single slice in a particular location and under a particular viewing angle of this complex 3D structure. So considering volume only by means of a set of planes leads to the loss of information. Moreover, in case of human bone texture images, the resolution of various imaging modalities is still low compared to the thickness of trabeculae. On routine clinical images, only the largest trabeculae can generally be distinguished.

The aim of this work is to know if the evaluation of the fractal dimension of a trabecular volume can be correctly obtained only from the analysis of planes (slices or projections) or it is indispensable to study the whole volume reconstructed from these slices.

An analysis of a 3D isotropic continuous fractional Brownian motion (fBm) process has been recently suggested [8]. This study has established a formal link between S3D (the self-similarity parameter of trabecular bone) and S2D (the self-similarity of its $2 \mathbf{D}$ projection onto the three orthogonal directions) that is $\mathbf{S} 2 \mathbf{D}=\mathrm{S} 3 \mathbf{D}+0.5$. In our paper, we extend the work of [8] and we study the relationship between fractal dimension 3D of a set and those of its projections and sections in every direction. Indeed, the result of [8] has been proved for a particular self-similar model and only for three particular projections on the Cartesian coordinate planes. This question arises because of the practical conditions under which some medical images are acquired (according non orthogonal planes). In addition, anisotropic features of trabecular tissues [10] lead to consider a self-affine character rather than a self-similar one.

This paper is organized as follows. After some recalls, we study in section 3 the behaviour of the Minkowski contents of projections of sets and the relations between Hausdorff measures and Minkowski contents under projection-type mappings and related topics. We give also examples illustrating the non stability of Minkowski dimensions of projected sets. In fact, they depend on 
the local properties of the set. For this aim we consider a large class of self-similar sets and especially Cantor sets. Section 4 is dedicated to the study of a relationship between the dimension of a set and those of its slices and we prove that for Suslin sets they are different and take account of local properties of the concerned set. Finally and as an application to the analysis of $3 \mathbf{D}$ and 2D bone structure, we use images obtained in clinical routine (CT scan images). We construct a volume of trabecular bone from these images and we calculate their 3D Minkowski dimension then we measure the 2D Minkowski dimension of their projections and slices. Projections are simulated by summation of voxels of the $3 \mathbf{D}$ volume. Slices are obtained from the same volume. In addition self-affine sets are used to modelize these volumes. We prove statistically that the relation between 3D and 2D structure depends on projections and slices. As a consequence it appears that a 3D fractal analysis is necessary to study the structure of the bone and to discriminate osteoporosis cases.

\section{Preliminaries}

Before giving our results and applications, let's recall some definitions. To be more convenient, we restrict our results and definitions to the 3 -dimensional space $\mathbb{R}^{3}$. For $n \in\{2,3\}$, the Lebesgue measure in $\mathbb{R}^{n}$ is denoted by $\mathcal{L}^{n}$. For any $x \in \mathbb{R}^{3}, 0<r<\infty, B(x, r)$ is the closed ball with centre $x$ and radius $r$.

For $0 \leq s \leq 3$, the $s$-dimensional Hausdorff measure of $A \subset \mathbb{R}^{3}$ is defined by

$$
\mathcal{H}^{s}(A)=\lim _{\varepsilon \longrightarrow 0} \inf \left\{\sum_{i} d\left(A_{i}\right)^{s}: A \subset \bigcup_{i=1}^{\infty} A_{i}, d\left(A_{i}\right) \leq \varepsilon\right\}
$$

where $d\left(A_{i}\right)$ is the diameter of $A_{i}$.

It is easily seen that each set $A$ has a unique "correct" value of the dimension parameter $s$ associated with it; i.e there exists a unique value, $\operatorname{dim}_{H} A$, of $s$ for which the Hausdorff measure $\mathcal{H}^{s}(A)$ "drops" from infinity to zero [4], i.e

$$
\mathcal{H}^{s}(A)=\left\{\begin{array}{cc}
\infty & \text { for } \quad s<\operatorname{dim}_{H} A \\
0 & \text { for } \quad s>\operatorname{dim}_{H} A
\end{array}\right.
$$

The number $\operatorname{dim}_{H} A$ is the Hausdorff dimension of $\mathrm{A}$. by

Let $A$ be a non-empty bounded subset of $\mathbb{R}^{3}$, the $s$-dimensional upper content of $A$ is defined

$$
\mathcal{M}^{s}(A)=\limsup _{\varepsilon \rightarrow 0}(2 \varepsilon)^{s-3} \mathcal{H}^{3}(A(\varepsilon))
$$

Where $A(\varepsilon)$ denotes the closed $\varepsilon$-neighbourhood of $A$ that is

$$
A(\varepsilon)=\left\{x \in \mathbb{R}^{3}: \inf |x-y| \leq \varepsilon\right\}
$$

It is known that [15] the Minkowski dimension is related to the upper Minkowski content by $\operatorname{dim} A=\inf \left\{s: \mathcal{M}^{s}(A)=0\right\}=\sup \left\{s: \mathcal{M}^{s}(A)>0\right\}$. 
Notice that for all $A \subset \mathbb{R}^{3}$, $\operatorname{dim}_{H} A \leq \operatorname{dim} A$ (see [4]) and this inequality can be strict with equality if $A$ is a self-similar set satisfying the "Open Set Condition" (OSC). Recall that self-similar sets are compact sets $K$ that satisfy $K=\cup_{i} S_{i} K$ for some finite collection of contracting similitudes $\left\{S_{i}\right\}_{1 \leq i \leq l}$ are well understood under separation conditions, such as the "Open Set Condition" [7]. The iterated function schemes (I.F.S) $\left\{S_{i}\right\}_{1 \leq i \leq l}$ are said to satisfy the OSC if there exists a nonempty open set $U$ such that $S_{i} U$ are disjoint and lie in $U$ for $i=1, \ldots, l$.

Consider a self-similar set $K \subset \mathbb{R}^{3}$ defined as the unique non-empty compact satisfying :

$$
\left.K=\bigcup_{i=1}^{m}\left(r_{i} K+b_{i}\right) \text { with } r_{i} \in\right] 0,1\left[\text { and } b_{i} \in \mathbb{R}^{3} .\right.
$$

We assume that the similitudes $S_{i}(x)=r_{i} x+b_{i}$ for $i=1, \ldots, l$ satisfy the OSC. It is well known that the Hausdorff and Minkowski dimensions coincide and are equal to the similarity dimension defined by $\sum_{i=1}^{l} r_{i}^{s}=1$, and the $s$-dimensional Hausdorff measure $\mathcal{H}^{s}(K)$ is positive and finite. But much less is known about the behaviour of Minkowski contents on general self-similar sets.

\section{Projections Results}

In this section, we study the relationship between the Minkowski dimension of a set and that of its projections using as examples two large classes of Suslin sets which are self-similar and self-affine sets. We also try to understand the behaviour of Hausdorff measure and Minkowski contents under projection-type mappings and related topics.

For a plane $V$ of $\mathbb{R}^{3}$ we note $V^{\perp}$ the orthogonal complement of $\mathrm{V}$ for $x \in \mathbb{R}^{3}$ there exist unique vectors $x_{V} \in V$ and $x_{V^{\perp}} \in V^{\perp}$ such that $x=x_{V}+x_{V^{\perp}}$. We let $p_{V}$ denote the orthogonal projection onto $V$ defined by $p_{V}(x)=x_{V}$ for $x \in \mathbb{R}^{3}$. Let $E \subseteq \mathbb{R}^{3}$ be a Borel set. It has been proved that for almost all planes $V$ of $\mathbb{R}^{3}$ we have $\operatorname{dim}_{H}\left(p_{V} E\right)=\min \left(\operatorname{dim}_{H} E, 2\right)$. This result was first obtained in the planer case by Marstrand [12] in 1954 and later generalized to arbitrary dimensions by Mattila [13]. Unfortunately the Minkowski dimension of the projection of a Borel set does not satisfy a similar formula. In view of the dual role of the Hausdorff measure/Hausdorff dimension and Minkowski content/Minkowski dimension displayed in $\operatorname{dim}_{\mathrm{H}} A \leq \operatorname{dim} A$, this may seem rather surprising. Indeed, there is no simple formula for the Minkowski dimension of the projection of a Borel set. Falconer and Howroyd [5] proved that:

$$
\frac{\operatorname{dim} E}{1+\frac{1}{6} \operatorname{dim} E} \leq \operatorname{dim} p_{V} E \leq \min (\operatorname{dim} E, 2)
$$

for almost all planes $V$ of $\mathbb{R}^{3}$. These inequalities are the best possible.

As a continuity of these results we study the behaviour of the Minkowski dimensions, Hausdorff measure and Minkowski contents of projected sets and we illustrate with examples the instability of the Minkowski dimension of projections of sets.

We denote by $G(3,2)$ the Grassmannian manifold of 2-dimensional linear subspaces of $\mathbb{R}^{3}$. Let $\gamma_{3,2}$ be the orthogonally invariant probability measure on $G(3,2)$ then $\gamma_{3,2}$ can be reduced 
to the normalized surface measure on the unite sphere $S^{2}=\{x:|x|=1\}$. More precisely we shall introduce a measure on the so-called Grassmannian manifold $G(3,2)$ of planes of $\mathbb{R}^{3}$ by $\gamma_{3,2}(A)=\sigma^{2}\left(\underset{V \in A}{\cup} V^{\perp} \cap S^{2}\right), A \subset G(3,2)$ where $\sigma^{2}$ is the surface measure on $S^{2}$.

Let $V \in G(3,2)$ and $0<s<2$, we define the $s$-dimensional upper Minkowski content in $V$ for any $A \subset V$ by

$$
\mathcal{M}_{V}^{s}(A)=\limsup _{\varepsilon \rightarrow 0} \varepsilon^{s-2} \mathcal{H}^{2}(A(\varepsilon) \cap V)
$$

For $s=2$, we set

$$
\mathcal{M}_{V}^{s}(A)=\limsup _{\varepsilon \rightarrow 0}(\log \varepsilon)^{-1} \mathcal{H}^{2}(A(\varepsilon) \cap V)
$$

For more details see [15].

As we mentioned before, the aim of this work is to study the fractal analysis of a set $K$ in $\mathbb{R}^{3}$ and that of its projections on every plane. Our basic problem is: What can we say about the relations between the Minkowski contents, Hausdorff measures and dimensions of the projections $p_{V} K, V \in G(3,2)$ and those of $K$ ? One direction is clear, as $p_{V}$ is a Lipschitz map; $\left|p_{V} x-p_{V} y\right| \leq|x-y|$, we always have $\mathcal{M}^{s}\left(p_{V} K\right) \leq \mathcal{M}^{s}(K)$ and $\operatorname{dim} p_{V} K \leq \operatorname{dim} K$.

To give an idea about this problem let us look at some examples.

Cantor sets: $K_{d}$ For $0<d \leq \frac{1}{2}$, let $K_{d}$ be the "Eight Corner Cantor Set".

$$
K_{d}=\bigcap_{k=1}^{\infty} V_{k}^{d}, \quad V_{k}^{d}=\bigcup_{i=1}^{8^{k}} C_{k, i}
$$

Where each $C_{k, i}$ is a closed cube of side-length $d^{k}$ and they are defined as follows. First the $C_{1, i} \mathrm{~s}$ are the eight corners of the unit cube $[0,1] \times[0,1] \times[0,1]$ which are $\prod_{i=1}^{3}\left[\varepsilon_{i}(1-d), \varepsilon_{i}(1-d)+d\right]$ for all $\left(\varepsilon_{1}, \varepsilon_{2}, \varepsilon_{3}\right) \in\{0,1\}^{3}$.

If the cubes $C_{k, i}, i=1, \ldots, 8^{k}$, have been constructed, the $C_{k+1, j}$ s are obtained in the same way inside and in the corners of the $C_{k, i}$ s. Notice that the Cantor $K_{d}$ is self-similar (2.5).

The Cantor $K_{d}$ has $s_{d}=\frac{\log 8}{\log \frac{1}{d}}$ as fractal dimension. Indeed, in the $k$-th step $V_{k}^{d}$ is covered by $8^{k}$ cubes of diameter $\sqrt{3} d^{k}$. Moreover, one has $0<\mathcal{H}^{s_{d}}\left(K_{d}\right)<\infty$. For any $V \in G(3,2)$, the orthogonal projection of $K$ on the plane $V$ denoted by $p_{V} K$ is self similar (2.5). When we look at the projections $p_{V} K_{d}$, we immediately notice that when we project into the Cartesian coordinate planes, we get Cantor sets whose dimensions are $\frac{\log 4}{\log \frac{1}{d}}=\frac{2}{3} s_{d}$.

Looking carefully at these projections on different planes $V$ we find a countable dense set of planes $V$ for which $p_{V} K_{d}$ is a self-similar set with fractal dimension strictly less than $s_{d}$. This happens often when $p_{V}$ maps two different cubes $C_{k, i}$ exactly onto the same shaped regular quadrilateral. However, taking into account a result of Marstrand [12], we shall see that this behaviour is exceptional. 
In fact, one has,

$$
\text { If } s_{d} \leq 2, \operatorname{dim}\left(p_{V} K_{d}\right)=s_{d} \text { for } \gamma_{3,2}-\text { almost all } V \in G(3,2) .
$$

and

$$
\text { If } s_{d}>2, \mathcal{L}^{2}\left(p_{V} K_{d}\right)>0 \text { for } \gamma_{3,2} \text {-almost all } V \in G(3,2) .
$$

More is said about these exceptional sets in the following theorem.

Theorem 1. Let $0<s \leq 2$ and $A$ be a Borel Suslin set with $0<\mathcal{H}^{s}(A)<\infty$ then $\mathcal{M}_{V}^{s}\left(p_{V} A\right)>0$ for $\gamma_{3,2}$-almost every $V \in G(3,2)$ and

$$
\int \mathcal{M}_{V}^{s}\left(p_{V} A\right) d \gamma_{3,2} V \geq c \mathcal{H}^{s}(A)
$$

where $c$ is a positive constant depending on $s$ and $A$.

Moreover,

$$
\operatorname{dim}_{H}\left\{V \in G(3,2): \mathcal{M}_{V}^{s}\left(p_{V}(A)\right)=0\right\} \leq s .
$$

Before giving the proof of theorem 1 let us give this result as stated by Mattila [15].

Proposition 2. Let $0<s \leq 2$ and $A \subset \mathbb{R}^{3}$ be a Borel set. Let $P \subset G(3,2)$ be a Borel set. If there exists a Borel probability measure $\mu$ compactly supported in $\mathbb{R}^{3}$ such that for some constant $b, \mu(B(x, \varepsilon)) \leq b \varepsilon^{s}$ for $x \in \mathbb{R}^{3}$ and $\varepsilon>0$, then for $0<\varepsilon<\frac{1}{2}$ one has,

$$
\int_{P} \mathcal{H}^{2}\left(p_{V} A(\varepsilon)\right) d \gamma_{3,2} V \geq c(s) b^{-1} \gamma_{3,2}(P)^{2} \varepsilon^{2-s}, \text { if } 0<s<2
$$

and

$$
\int_{P} \mathcal{H}^{2}\left(p_{V} A(\varepsilon)\right) d \gamma_{3,2} V \geq c b^{-1} \gamma_{3,2}(P)^{2}\left(\log \left(\frac{1}{\varepsilon}\right)\right)^{-1}, \text { if } s=2
$$

Now, let us prove theorem 1

Proof. Let $0<s<2$, the case $s=2$ is proved similarly.

Since $0<\mathcal{H}^{s}(A)<\infty$, using Frostman's Lemma [2] we find a Borel probability measure $\mu$ supported in $A$ and for $x \in \mathbb{R}^{3}, 0<\varepsilon<\infty ; \mu(B(x, \varepsilon)) \leq b \varepsilon^{s}$ with $b=c^{\prime}\left(\mathcal{H}^{s}(A)\right)^{-1}$ where $c^{\prime}$ is a constant.

Let $P \subset G(3,2)$ be a Borel set such that $\mathcal{M}_{V}^{s}\left(p_{V} A\right)<\infty$ for $V \in P$. By Egoroff's theorem, there exists a Borel set $Q \subset P$ such that $\gamma_{3,2}(Q) \geq \frac{1}{2} \gamma_{3,2}(P)$

and $\sup _{0<\varepsilon<\delta} \varepsilon^{s-2} \mathcal{H}^{2}\left(p_{V} A(\varepsilon)\right)$ converges uniformly in $Q$ as $\delta \searrow 0$.

Due to the previous proposition we have for $\varepsilon>0$,

$$
\varepsilon^{s-2} \int_{Q} \mathcal{H}^{2}\left(p_{V} A(\varepsilon)\right) d \gamma_{3,2} V \geq c_{1} b^{-1} \gamma_{3,2}(P)^{2}
$$

Hence, 


$$
\begin{aligned}
\int_{P} \mathcal{M}_{V}^{s}\left(p_{V} A\right) d \gamma_{3,2} V & \geq \lim _{\delta \rightarrow 0} \int_{Q} \sup _{0<\varepsilon<\delta} \varepsilon^{s-2} \mathcal{H}^{2}\left(p_{V} A(\varepsilon)\right) d \gamma_{3,2} V \\
& \geq \lim _{\delta \rightarrow 0} \sup _{0<\varepsilon<\delta} \int_{Q} \varepsilon^{s-2} \mathcal{H}^{2}\left(p_{V} A(\varepsilon)\right) d \gamma_{3,2} V \\
& \geq c_{1} b^{-1} \gamma_{3,2}(P)^{2} \geq c \mathcal{H}^{s}(A) \gamma_{3,2}(P)^{2}
\end{aligned}
$$

Which proves the theorem.

Remark 3. 1. Let us remark that in the case of self-similar sets, the Minkowski content is equivalent to the Hausdorff measure and the hypothesis of theorem 1 can be changed by $\mathcal{M}^{s}(K)>0$.

2. Notice that if $\mathcal{H}^{s}(K)>0$ we do not necessarily have $\mathcal{H}^{s}\left(p_{V} K\right)>0$ even almost every where. Indeed we can construct a Suslin set $E$ of $\mathbb{R}^{3}$ such that $\mathcal{H}^{s}\left(p_{V} E\right)=0$ for all $V \in$ $G(3,2)$, for $s<2$ (see example 1$)$.

3. Under the hypothesis of theorem 1, we can deduce that if $\operatorname{dim}_{H} K=s$ then $\operatorname{dim} p_{V}(K) \geq s$ for $\gamma_{3,2}$-almost all $V \in G(3,2)$.

Example 4. For $0<s<2$ there is a compact set $E \subset \mathbb{R}^{3}$ such that $\mathcal{H}^{s}(E)>0$ and $\mathcal{H}^{s}\left(p_{L} E\right)=0$ for all $L \in G(3,2)$.

Let $Q$ be a cube in $\mathbb{R}^{3}$ with sides parallel to the Cartesian coordinate planes denoted xoy, yoz and $x o z$ with side-length $d$ and center $(a, b, c)$. Given positive integers $m, n$ and $p$ and $\Theta \in\left[0, \frac{\pi}{2}\right]$ we first describe an operation to generate a disjoint collection of subcubes of $Q$.

Let $R$ be the parallelepiped $\left\{(x, y, z):|x-a| \leq \frac{d}{2},|y-b| \leq \frac{d}{2 n},|z-c| \leq \frac{d}{2},\right\}$ (Figure 1) contained in $Q$. Divide $R$ into cubes $Q_{1}^{\prime}, \ldots, Q_{n}^{\prime}$ of side-length $\frac{d}{n}$. Let $Q_{i}$ be the cube with the same center as $Q_{i}^{\prime}$ and with side length $\frac{d}{2 n}$ having 2 sides making an angle $\Theta$ with the xoy plane. Partition each $Q_{i}$ into $m^{3}$ cubes $P_{i j}^{\prime}$ of side-length $\frac{d}{2 m n}$. Finally let $P_{i j}$ be the cube with sides parallel to the coordinate planes which has the same center as $P_{i j}^{\prime}$ and side-length $d^{\prime}$ with $d^{\prime}$ is defined by $m^{3} n d^{\prime s}=d^{s}$. We define $\alpha=\alpha(m, n)=\arctan \left(\frac{d^{\prime}}{d / 2 n}\right)$. Let $V_{\Phi}$ be the plane having an angle $\Phi$ with the xoy plane where $\Theta \leq \Phi \leq \Theta+\alpha$ or $\Theta+\frac{\pi}{2} \leq \Phi \leq \Theta+\frac{\pi}{2}+\alpha$ then there are quadrilaterals $H_{i k} \subset V_{\Phi}, k=1, \ldots, m ; i=1, \ldots, n$ such that $p_{V_{\Phi}}\left(\cup_{j=1}^{m^{3}} P_{i j}\right) \subset \bigcup_{k=1}^{2 m^{2}} H_{i k}$ with $d\left(H_{i k}\right)=2 \sqrt{3} d^{\prime}$, so $\sum_{i, k} d\left(H_{i k}\right)^{s}=\frac{(2 \sqrt{3} d)^{s}}{m^{2}}$. Let us now choose strictly increasing sequences $m_{k}$ and $n_{k}$ of positive integers and let $\alpha_{k}=\alpha\left(m_{k}, n_{k}\right)$. Starting from the cube $Q_{1,1}=\left[0, d_{1}\right] \times\left[0, d_{1}\right] \times\left[0, d_{1}\right]$, perform the above operation with $m=m_{1}, n=n_{1}, \Theta=0$. In each cube $P_{i j}$ thus obtained perform the operation with $m=m_{1}, n=n_{1}, \Theta=\alpha_{1}$. Continue this with $m=m_{1}, n=n_{1}, \Theta=j \alpha_{1}$ for $j=2,3, \ldots$ until $\frac{\pi}{2} \leq j \alpha_{1}$. Let $Q_{2,1}, \ldots, Q_{2, p_{1}}$ be all the subcubes of $Q$ obtained at the least step. Their common side-length $d_{2}$ satisfies $p_{1} d_{2}^{s}=d_{1}^{s}$. Furthermore, for every $\Phi \in[0, \pi]$ there are quadrilaterals $H_{j} \subset V_{\Phi}$ such that 


$$
\begin{gathered}
P_{V_{\Phi}}\left(\cup_{i} Q_{2, i}\right) \subset \underset{j}{\cup} H_{j} \\
\sum_{j} d\left(H_{j}\right)^{s} \leq \frac{\left(2 d_{1}\right)^{s}}{m_{1}}
\end{gathered}
$$

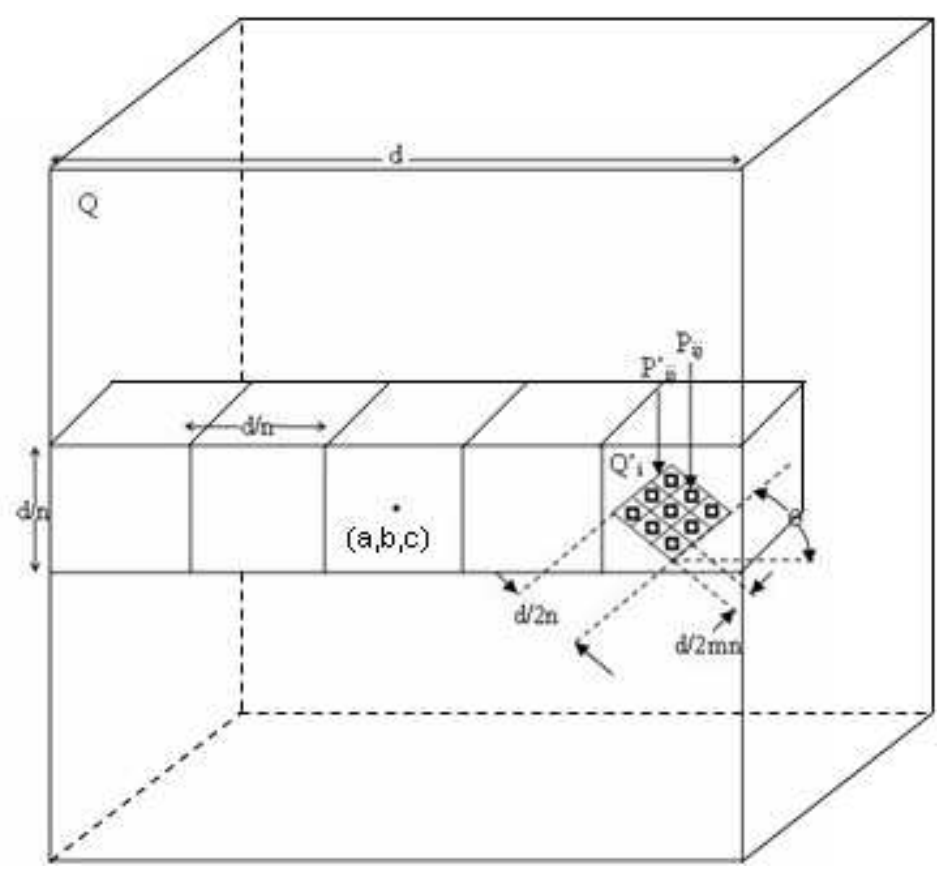

Figure 1: Example1

Next, in each cube $Q_{2, i}$ we perform the similar sequence of operations with parameters $m_{2}, n_{2}$, $j \alpha_{2}, j=0,1,2, \ldots$ until $j \alpha_{2} \geq \frac{\pi}{2}$ obtaining the cubes $Q_{3, j}$. Continuing in this process we get cubes $Q_{k, j}, j=1, \ldots p_{k}, k=1,2, \ldots$ and we define

$$
E=\bigcap_{k=1}^{\infty} \bigcup_{j=1}^{p_{k}} Q_{k, j}
$$

Then $0<\mathcal{H}^{s}(E)<\infty$, and relations similar to (3.14) imply that $\mathcal{H}^{s}\left(p_{V} E\right)=0$ for all $V \in G(3,2)$.

Now, let us consider the following class of self-similar sets that includes the "Eight Corner Cantor Set" $K_{d}$. 


$$
\Lambda=\left\{\sum_{n=0}^{\infty} r_{n} m^{-n} ; r_{n} \in\left\{b_{1}, \ldots, b_{2 m}\right\}\right\},
$$

where $b_{1}, \ldots, b_{2 m}$ are distinct vectors in $\mathbb{R}^{3}$ and $m \geq 2$ an integer, then we have the following result showing that these sets project to zero Lebesgue measure in almost all directions.

Theorem 5. Let $m \geq 2$, suppose that the parts $\Lambda_{i}=b_{i}+m^{-1} \Lambda, i=1, \ldots, 2 m$ are pairwise disjoint then

1. If $m=2$, one has $\mathcal{L}^{2}\left(p_{V} \Lambda\right)>0$ for all $V \in G(3,2)$.

2. If $m \geq 3$, one has $\mathcal{L}^{2}\left(p_{V} \Lambda\right)=0$ for $\gamma_{3,2}$-almost all $V \in G(3,2)$.

One can check that for $m=2$, the set $p_{V} \Lambda$ is a non degenerate quadrilateral having positive length expect three.

The proof of theorem 5 needs several lemmas dealing with subsets of $\mathbb{R}^{2}, K=\bigcup_{i=1}^{m^{2}}\left(\frac{1}{m} K+d_{i}\right)$. Let us first look more generally at this type of self-similar subsets of $\mathbb{R}^{2}$.

Consider a compact set $K$ of $\mathbb{R}^{2}$ such that for some integer $m \geq 3$ and some $d_{1}, \ldots, d_{m^{2}} \in$ $\mathbb{R}^{2}, K=\bigcup_{i=1}^{m^{2}} K_{i}$ with $K_{i}=\frac{1}{m} K+d_{i}$.

Lemma 6. 1. $\mathcal{L}^{2}\left(K_{i} \cap K_{j}\right)=0$ for $i \neq j$.

2. $K_{i} \cap K_{j} \neq \emptyset$ for some $i \neq j$.

For the proof see the appendix.

Before stating the next lemma we need to introduce some notations.

Since $K_{i}=\frac{1}{m} K+d_{i}=\frac{1}{m}\left(\bigcup_{j=1}^{m^{2}}\left(\frac{1}{m} K+d_{j}\right)\right)+d_{i}=\bigcup_{j=1}^{m^{2}} K_{i j}$, where $K_{i j}=\frac{1}{m^{2}} K+\frac{1}{m} d_{j}+d_{i}$, we can write $K$ also as the union of the $m^{4}$ sets $K_{i j}$. This operation can be iterated. For each positive integer $l$ the set $K$ is the union of $m^{2 l}$ pieces called cylinders of order $l$ each of which is a translate of $m^{-1} K$. Let $I=\left\{1,2, \ldots, m^{2}\right\}$ and $I^{l}=\left\{u=u_{1} \ldots u_{l}: u_{i} \in I\right\}, l=1,2, \ldots$

Then for each $l, K=\cup_{u \in I^{l}} K_{u}$ where $K_{u}=m^{-l} K+\sum_{p=1}^{l} d_{u_{p}} m^{-p+1}=K_{u_{1} \ldots u_{m}{ }^{2}}$.

Repeating the proof of Lemma 6 for this decomposition demonstrates that $\mathcal{L}^{2}\left(K_{u} \cap K_{v}\right)=0$ for different $u$ and $v$ in $I^{l}$.

The following notion has been established by Bandt and Graf [1].

Definition 7. Let $\varepsilon>0$, two cylinders $K_{u}$ and $K_{v}$ are $\varepsilon$-relatively close if $u$ and $v$ belong to $I^{l}$ for some $l$ and $K_{u}=K_{v}+x$ for some $x$ with $|x| \leq \varepsilon d\left(K_{u}\right)$.

Where $d\left(K_{u}\right)$ is the diameter of $K_{u}$.

Note that for $u, v \in I^{l}, K_{v}$ is always a translation of $K_{u}$, but we are now required not to translate too much. 
Lemma 8. If for every $\varepsilon>0$ there are $l$ and $u, v \in I^{l}$ with $u \neq v$ such that $K_{u}$ and $K_{v}$ are $\varepsilon$-relatively close then $\mathcal{L}^{2}(K)=0$.

For the proof see the appendix.

Let us prove now the theorem 5 that $\mathcal{L}^{2}\left(p_{V} \Lambda\right)=0$ for $\gamma_{3,2}$-almost all $V \in G(3,2)$.

Proof. Set $p_{V}(\Lambda)={ }^{V} \Lambda$ to make it more convenient to use the notations ${ }^{v} \Lambda_{u}$ from above. For $\varepsilon>0$, let $V_{\varepsilon}=\left\{V \in G(3,2): \exists l, u, v\right.$ such that $u, v \in I^{l}, u \neq v$ and ${ }^{v} \Lambda_{u}$ and ${ }^{v} \Lambda_{v}$ are $\varepsilon$-relatively close $\}$. It follows from lemma 8 and the definition of $G(3,2)$ (we can also handle $G(3,2)$ by identifying any hyperplane with its orthogonal complement) that it suffices to show that for every $\varepsilon>0, \gamma_{3,2}\left(G(3,2) \backslash V_{\varepsilon}\right)=0$. Let $\varepsilon>0$ and $V \in G(3,2)$, by lemma 6(2) ${ }^{V} \Lambda_{i} \cap^{V} \Lambda_{j} \neq \emptyset$ for some $i \neq j$ which implies that there are $x \in \Lambda_{i}$ and $y \in \Lambda_{j}$ such that $p_{V} x=p_{V} y$. Let $l>1$ be an integer then $x \in \Lambda_{u}$ and $y \in \Lambda_{v}$ for some $u, v \in I^{l}$ with $u \neq v$. Let $V_{0} \in G(3,2)$ such that $p_{V_{0}} \Lambda_{u}=p_{V_{0}} \Lambda_{v}$ (that is $p_{v_{0}}$ maps the cubes of side-length $m^{-l}$ which contain $\Lambda_{u}$ and $\Lambda_{v}$ onto the same quadrilateral). Then ${ }^{V_{0}} \Lambda_{u}$ and ${ }^{V_{0}} \Lambda_{v}$ are " 0 -relatively close" that is ${ }^{V_{0}} \Lambda_{u}={ }^{V_{0}} \Lambda_{v}$ and a simple geometric inspection shows that ${ }^{W} \Lambda_{u}$ and ${ }^{W} \Lambda_{v}$ are $\varepsilon$-relatively close when the angle between $V_{0}$ and $W$ is less then $b \varepsilon m^{-l}$ where $b$ is a constant. Thus, with another constant $c>0$, $G=\left\{W \in V_{\varepsilon}:\left(W^{\wedge}, V\right)<\mathrm{cm}^{-l}\right\}$ contains a set of measure $b \varepsilon m^{-l}$. Since this is true for every $l$, it follows that $\gamma_{3,2}\left(G(3,2) \backslash V_{\varepsilon}\right)=0$ as required.

In this section, we have shown using examples of Suslin sets which include two large classes of fractal sets (self-similar and self-affine sets) that, in general, there is no relationship between the Minkowski dimension of a set of $\mathbb{R}^{3}$ and that of its projections.

In the next section we will study if it is possible to find a relation between the Minkowski dimension of a set of $\mathbb{R}^{3}$ and those of its slices.

\section{Slices Results}

The Hausdorff dimensional properties of sections of sets that are intersections of sets and planes have been known for many years. They follow the "rule of adding codimension" for intersections of "classical" rectifiable or smooth sets. Let $V$ be a plane of $\mathbb{R}^{3}$ and $V^{\perp}$ is the orthogonal complement of $V$, for $a \in V^{\perp}$ we denote by $V_{a}$ the plane $\{v+a: v \in V\}$ the a-translate of $V$.

It has been proved by [13] that for all $E \subset \mathbb{R}^{3}$ and $V \in G(3,2), \operatorname{dim}_{H}\left(E \cap V_{a}\right) \leq \max \left\{0, \operatorname{dim}_{H} E-\right.$ $1\}$ for $\mathcal{H}^{1}$ almost all $a \in V^{\perp}$ and if $s>1$ and $E$ is a Borel Suslin set of $\mathbb{R}^{3}$ with $0<\mathcal{H}^{s}(E)<\infty$ then $\operatorname{dim}_{H} E \cap V_{a}=s-1$ and $\mathcal{H}^{s-1}\left(E \cap V_{a}\right)<\infty$ for $\mathcal{H}^{s} \times \gamma_{3,2}$ almost all $(a, V) \in E \times G(3,2)$.

In this paper we are also interested in the Minkowski dimension properties of sections of sets and to a possible version of the previous result corresponding to the Minkowski dimension. Unfortunately, we do not have such results between Minkowski dimension of a set and of its sections. The geometry of these dimensions is much less regular than that of Hausdorff dimension. However, given a generalized Minkowski dimension $\operatorname{dim}_{p}^{V}$ for a given $V \in G(3,2)$ :

If $E$ is a set of $\mathbb{R}^{3}$

$$
\operatorname{dim}_{p}^{V} E=\inf \left\{\sup _{i} \operatorname{dim}^{V} E_{i}: E \subset \bigcup_{i=1}^{\infty} E_{i} \text { and } E_{i} \text { is bounded for all } i\right\}
$$


and for a bounded set $A \subset \mathbb{R}^{3}$

$$
\operatorname{dim}^{V} A=\max \left\{0, \limsup _{k \rightarrow \infty} \frac{\log N_{k}^{V}(A)}{\log 2^{k}}\right\}
$$

Where

$E$

$N_{k}^{V}(A)=\sum_{i} \mathcal{H}^{1}\left(p_{V^{\perp}}\left(A \cap Q_{i}^{k}\right)\right)$ for $V \in G(3,2),\left(Q_{i}^{k}\right)$ are the $2^{-k}$-mesh cubes that intersect

and $p_{V^{\perp}}: \mathbb{R}^{3} \rightarrow V^{\perp}$ is the orthogonal projection onto the orthogonal complement $V^{\perp}$ of $V$.

We obtain the following result.

Theorem 9. Let E be a compact set of $\mathbb{R}^{3}$ then one has,

$$
\operatorname{dim}\left(E \cap V_{a}\right)=\operatorname{dim}_{p}^{V} E \text { for } \mathcal{H}^{1}-\text { almost all } a \in V^{\perp},
$$

Remark 10. 1. Before giving the proof of the previous theorem, notice that unlike what has been established in [13] concerning the relationship between Hausdorff dimensions of a given set and those of its sections, our results show that such relationship does not exist in the case of the Minkowski dimensions. In fact, the weights attached to the balls used in defining the measures and dimensions are modified to take account of local projection properties of the set concerned and the relation given by theorem 9 proves that the Minkowski dimension of a section depends on the local properties of this set.

2. In the case of a self-similar set $E$ one has $\operatorname{dim}_{p}^{V} E=\operatorname{dim} E-1$ for $\gamma_{3,2}$-almost all $V \in$ $G(3,2)$.

Proof. Let us prove that $\operatorname{dim}\left(E \cap V_{a}\right) \leq \operatorname{dim}_{p}^{V} E$ for $\mathcal{H}^{1}$-almost all $a \in V^{\perp}$.

For a given $\varepsilon>0$, there are bounded sets $E_{1}, E_{2}, \ldots, E_{l}$ with $E \subset \cup_{i=1}^{l} E_{i}$ and $\operatorname{dim}^{V} E_{i} \leq$ $\operatorname{dim}_{p}^{V} E+\varepsilon$. For $1 \leq i \leq l$ and $k=1,2, \ldots$, let $\left(Q_{i j}^{k}\right)$ be the $2^{-k}$-mesh cubes determined by a coordinate system with $V$ and $V^{\perp}$ as coordinate planes and let $\chi_{p_{V^{\perp}}\left(E_{i} \cap Q_{i j}^{k}\right)}$ be the characteristic function of the set $p_{V \perp}\left(E_{i} \cap Q_{i j}^{k}\right)$.

Since $N_{k}\left(E \cap V_{a}\right) \leq \sum_{i} \sum_{j} \chi_{p_{V^{\perp}}\left(E_{i} \cap Q_{i j}^{k}\right)}(a)$ we have

$$
\begin{aligned}
\int_{V^{\perp}}^{*} N_{k}\left(E \cap V_{a}\right) d \mathcal{H}^{1} a & \leq \sum_{i} \sum_{j} \int_{V^{\perp}}^{*} \chi_{p_{V^{\perp}}\left(E \cap Q_{j}^{k}\right)}(a) d \mathcal{H}^{1} a \\
& \leq \sum_{i} \sum_{j} \mathcal{H}^{1}\left(p_{V^{\perp}}\left(E \cap Q_{i j}^{k}\right)\right)=\sum_{i} N_{k}^{V}\left(E_{i}\right)
\end{aligned}
$$

Where $\int^{*}$ denotes the upper integral.

If $s>\operatorname{dim}_{p}^{V} E$, then for all $i, N_{k}^{V}\left(E_{i}\right) \leq 2^{k(s+\varepsilon)-i}$ for $k$ sufficiently great. 
So,

$$
\begin{aligned}
\int_{V^{\perp}}^{*} \sum_{k} 2^{-k(s+2 \varepsilon)} N_{k}\left(E \cap V_{a}\right) d \mathcal{H}^{1} a & \leq \sum_{k} 2^{-k(s+2 \varepsilon)} \int_{V^{\perp}}^{*} N_{k}\left(E \cap V_{a}\right) d \mathcal{H}^{1} a \\
& \leq \sum_{k} 2^{-k(s+2 \varepsilon)} \sum_{i} 2^{k(s+\varepsilon)-i} \\
& \leq \sum_{i} 2^{-i} \sum_{k} 2^{-k \varepsilon}<\infty
\end{aligned}
$$

Giving $\lim _{k \rightarrow \infty} 2^{-k(s+2 \varepsilon)} N_{k}\left(E \cap V_{a}\right)=0$ for $\mathcal{H}^{1}-$ almost all $a \in V^{\perp}$.

Thus, $\operatorname{dim}\left(E \cap V_{a}\right) \leq s$ for $\mathcal{H}^{1}$-almost all $a \in V^{\perp}$ and (4.3) follows by taking a sequence $\left(s_{i}\right)$ tending to $\operatorname{dim}_{p}^{V} E$ from above.

Now let prove the second inequality. Suppose that $\operatorname{dim}\left(E \cap V_{a}\right)<s$ for $\mathcal{H}^{1}$-almost all $a \in V^{\perp}$.

For all $i=1,2, \ldots$,

define $A_{i}=\left\{a \in p_{V^{\perp}}(E): N_{k}\left(E \cap V_{a}\right) \leq i 2^{k s}\right.$ for all $\left.k=1,2, \ldots\right\}$

and $B_{i}=\left\{x \in E: p_{V^{\perp}}(x) \in A_{i}\right\}$.

Notice that $A_{i}$ is $\mathcal{H}^{1}$-measurable, since the function $a \longmapsto N_{k}\left(E \cap V_{a}\right)$ is $\mathcal{H}^{1}$-measurable.

Moreover,

$$
\mathcal{H}^{1}\left(p_{V^{\perp}}(E) \backslash \bigcup_{i=1}^{\infty} B_{i}\right)=\mathcal{H}^{1}\left(p_{V^{\perp}}(E) \backslash \bigcup_{i=1}^{\infty} A_{i}\right)=0
$$

For given $i=1,2, \ldots$, we have $p_{V^{\perp}}\left(B_{i} \cap Q_{j}^{k}\right)=p_{V^{\perp}}\left(E \cap Q_{j}^{k}\right) \cap A_{i}$ for all $k=1,2, \ldots$, where $Q_{j}^{k}$ are the $2^{-k}$-mesh cubes determined by a coordinate system with $V$ and $V^{\perp}$ as coordinate planes.

Hence,

$$
\begin{aligned}
N_{k}^{V}\left(B_{i}\right) & =\sum_{j} \mathcal{H}^{1}\left(p_{V^{\perp}}\left(E \cap Q_{j}^{k}\right) \cap A_{i}\right) \\
& =\sum_{j} \int \chi_{p_{V^{\perp}}\left(E \cap Q_{j}^{k}\right)}(a) \chi_{A_{i}} d \mathcal{H}^{1} a \\
& =\int_{A_{i}} N_{k}\left(E \cap V_{a}\right) d \mathcal{H}^{1} a \\
& \leq i 2^{k s} \mathcal{H}^{1}\left(A_{i}\right)
\end{aligned}
$$

Thus, $\operatorname{dim}^{V} B_{i} \leq s$ since $\mathcal{H}^{1}\left(A_{i}\right)<\infty$ due to the boundness of $E$. On the other hand, the decomposition $E=\bigcup_{i=1}^{\infty} B_{i} \cup\left(E \backslash \bigcup_{i=1}^{\infty} B_{i}\right)$ and (4.6) imply that $\operatorname{dim}^{V}\left(E \backslash \bigcup_{i=1}^{\infty} B_{i}\right)=0$. As a consequence, $\operatorname{dim}_{p}^{V} E \leq \sup _{i} \operatorname{dim}^{V} B_{i} \leq s$ which concludes the proof of the theorem.

Now we will give an example illustrating the instability of Minkowski dimensions of sections. We shall prove that there exist a compact set $E \subset \mathbb{R}^{3}$ and compact subsets $A$ and $B$ of $G(3,2)$ with $\gamma_{3,2}(A)>0$ and $\gamma_{3,2}(B)>0$ such that for all $V \in A$ we have $\mathcal{H}^{1}\left(p_{V^{\perp}} E\right)=0$ that is $E \cap V_{a}=\emptyset$ for $\mathcal{H}^{1}$-almost all $a \in V^{\perp}$ and for all $V \in B$ we have $\operatorname{dim}\left(E \cap V_{a}\right)=2$ for points $a$ in a non-empty open subset of $V$. 
Theorem 11. There exist compact sets $E \subset \mathbb{R}^{3}$ and $A, B \subset G(3,2)$ with $\gamma_{3,2}(A)>0$ and $\gamma_{3,2}(B)>0$ such that

1. For all $V \in A$, one has $\mathcal{H}^{1}\left(p_{V^{\perp}} E\right)=0$.

2. For all $V \in B$, there exists a non empty open subset $U_{V}$ of $V^{\perp} \operatorname{such}$ that $\operatorname{dim}\left(E \cap V_{a}\right)=2$ for all $a \in U_{V}$.

Before giving the proof of this theorem let us give some notations that we shall need for our construction.

Let $P \subset[0,1]^{3}$ be a non-degenerate closed parallelepiped. We name the edges of $P$ such that the shortest parallel edges are called 1-edges, the second shortest parallel edges are 2-edges and the third parallel edges are 3-edges. Notice that this numbering distinguishes edges which are not parallel; that is if two edges have the same length and not parallel then they have different numbers. We call

- $P_{1}^{1}$ and $P_{1}^{2}$ the 2 -faces of $P$ which are generated by the edges numbered by 2 and 3 .

- $P_{2}^{1}$ and $P_{2}^{2}$ the 2-faces of $P$ which are generated by the edges numbered by 1 and 3 .

- $P_{3}^{1}$ and $P_{3}^{2}$ the 2 -faces of $P$ which are generated by the edges numbered by 1 and 2 .

Let $\left(e_{1}, e_{2}, e_{3}\right)$ be the standard basis of $\mathbb{R}^{3}$. For $i=1,2,3$ we let $w_{1}, w_{2}, w_{3}$ be the planes spanned respectively by $\left\{e_{2}, e_{3}\right\},\left\{e_{1}, e_{3}\right\}$ and $\left\{e_{1}, e_{2}\right\}$.We call $P \subset[0,1]^{3}$ a hyperregular parallelepiped in $\mathbb{R}^{3}$ if $P_{i}^{1}$ and $P_{i}^{2}$ are parallel to $w_{i}$ for $i \neq 2$; let $P_{i}^{1}$ be the one that is nearest to $w_{i}$. For a hyperregular parallelepiped $P$ and $\delta>0$ we define

$$
A_{3,2}(P)=\left\{\begin{array}{c}
V: V \text { is an affine plane meeting both } P_{i}^{1} \text { and } P_{i}^{2} \\
\text { for all } i \neq 3 \text { but not } P_{3}^{1} \text { and } P_{3}^{2}
\end{array}\right\}
$$

and

$$
A_{3,2}^{\delta}(P)=\left\{V \in A_{3,2}(P): \operatorname{dist}\left(V \cap P, P_{3}^{2}\right) \geq \delta\right\}
$$

where $\operatorname{dist}\left(V \cap P, P_{3}^{2}\right)=\inf \left\{|a-b|: a \in V \cap P, b \in P_{3}^{2}\right\}$ is the distance between $V \cap P$ and $P_{3}^{2}$.

We can have the same definitions in the plane with similar notations. Let us quote a lemma of Mattila [14] describing the plane case.

Lemma 12. There are disjoint compact sets $A, B \subset G(2,1)$ with $\gamma_{2,1}(A)>0$ and $\gamma_{2,1}(B)>0$ such that for all hyperregular parallelograms $P \subset[0,1]^{2}$ and for all $\varepsilon>0$ there exists a finite family $P_{\varepsilon}$ of hyperregular subparallelograms of $P$ with the following properties:

1. $\mathcal{H}^{1}\left(p_{L^{\perp}}\left(\cup P_{\varepsilon}\right)\right) \leq \varepsilon$ for all $L \in A$.

2. There is $\delta>0$ such that if $L \in A_{2,1}(P) \cap A_{2,1}\left([0,1]^{2}\right)$ is parallel to some line belonging to $B$ then there exists $Q \in P_{\varepsilon}$ such that $L \in A_{2,1}^{\delta}(Q)$. 
The following lemma provides the higher-dimensional version of the previous one.

Lemma 13. There are disjoint compact subsets $A, B \subset G(3,2)$ with $\gamma_{3,2}(A)>0$ and $\gamma_{3,2}(B)>0$ such that for all hyperregular parallelepipeds $P \subset[0,1]^{3}$ and for all $\varepsilon>0$ there exists a finite family $P_{\varepsilon}$ of hyperregular subparallelepipeds of $P$ with the following properties:

1. $\mathcal{H}^{1}\left(p_{V^{\perp}}\left(\cup P_{\varepsilon}\right)\right) \leq \varepsilon \quad$ for all $V \in A$.

2. There is $\delta>0$ such that if $V \in A_{3,2}(P) \cap A_{3,2}\left([0,1]^{3}\right)$ is parallel to some plane belonging to $B$ then there exists $Q \in P_{\varepsilon}$ such that $V \in A_{3,2}^{\delta}(Q)$.

For the proof of lemma 13 see the appendix.

Now let us prove the theorem 11.

Proof. Let $A, B \subset G(3,2)$ be as in lemma 13. We use the notation $P_{1,1}=[0,1]^{3}$, using lemma 13 we find hyperregular parallelepipeds $Q_{2,1}, \ldots, Q_{2, l_{2}} \subset P_{1,1}$ such that for all $V \in A$,

$$
\mathcal{H}^{1}\left(p_{V^{\perp}}\left(\bigcup_{q=1}^{l_{2}} Q_{2, q}\right)\right) \leq \frac{1}{2}
$$

Further, if $V \subset A_{3,2}\left(P_{1,1}\right)$ is parallel to some plane belonging to $B$ then $V \in A_{3,2}\left(Q_{2, q}\right)$ for some $1 \leq q \leq l_{2}$. For all $1 \leq q \leq l_{2}$ and $i=1,2$ let $d_{i}\left(Q_{2, q}\right)$ be the length of the $i$-edges of $Q_{2, q}$. Let $k_{2}$ be the smallest positive integer such that for all $1 \leq q \leq l_{2}, k_{2} \geq d_{1}\left(Q_{2, q}\right)^{-3}$. Dividing each $Q_{2, q}$ into $k_{2}^{2}$ hyperregular parallelepipeds with all the edges parallel to the corresponding edges of $Q_{2, q}$ and with the length of the $i$-edges equal to $\frac{1}{k_{2}} d_{i}\left(Q_{2, q}\right)$ for $i=1,2$, we obtain hyperregular parallelepipeds $P_{2,1}, \ldots, P_{2, N_{2}}$ where $N_{2}=l_{2}\left(k_{2}\right)^{2}$. It is easy to see that $\mathcal{H}^{1}\left(p_{V^{\perp}}\left(\cup_{q=1}^{N_{2}} P_{2, q}\right)\right) \leq \frac{1}{2}$ for all $V \in A$. By lemma 13 we find for all $1 \leq q \leq N_{2}$, hyperregular parallelepipeds $Q_{3,1}^{q}, \ldots, Q_{3, l_{3}^{q}}^{q} \subset$ $P_{2, q}$ such that for all $V \in A$,

$$
\mathcal{H}^{1}\left(p_{V^{\perp}}\left(\cup_{p=1}^{l_{3}^{q}} Q_{3, p}^{q}\right)\right) \leq \frac{1}{3 N_{2}}
$$

Moreover, if $V \in A_{3,2}\left(P_{2, q}\right) \cap A_{3,2}\left(P_{1,1}\right)$ is a plane parallel to some plane belonging to $B$, there exists $1 \leq p \leq l_{3}^{q}$ such that $V \in A_{3,2}\left(Q_{3, p}^{q}\right)$. As done before we repeat the same process. Let $k_{3}$ be the smallest integer such that for all $1 \leq q \leq N_{2}$ and $1 \leq p \leq l_{3}^{q}$ we have $k_{3} \geq$ $d_{1}\left(Q_{3, p}^{q}\right)^{-5}$, then there exist hyperregular parallelepipeds $P_{3,1}, \ldots, P_{3, N_{3}}$ where $N_{3}=\sum_{q=1}^{N_{2}} l_{3}^{q}\left(k_{3}\right)^{2}$ and $\mathcal{H}^{1}\left(p_{V^{\perp}}\left(\bigcup_{q=1}^{N_{3}} P_{3, q}\right)\right) \leq \frac{1}{3}$

Continuing in this way we obtain a compact set $E=\bigcap_{p=1}^{\infty} \bigcup_{q=1}^{N_{p}} P_{p, q}$ satisfying for all $V \in A$, and positive integers $p$

$$
\mathcal{H}^{1}\left(p_{V^{\perp}}(E)\right) \leq \mathcal{H}^{1}\left(p_{V^{\perp}}\left(\bigcup_{q=1}^{N_{p}} P_{p, q}\right)\right) \leq \frac{1}{p}
$$

which proves the first claim. 
Let $V \in A_{3,2}\left(P_{1,1}\right)$ be parallel to some plane belonging to $B$. By the construction for all $j$ we have $V \in A_{3,2}\left(Q_{j, p}^{q}\right)$ for some $1 \leq q \leq N_{j-1}$ and $1 \leq p \leq l_{j}^{q}$ and so $V \in A_{3,2}\left(P_{j, i}\right)$ for all $P_{j, i} \subset Q_{j, p}^{q}$. Therefore we need at least $\left(\frac{k_{j}}{3}\right)^{2}$-squares with side-length

$$
h_{j}=\frac{1}{k_{j}} \min _{1 \leq q \leq N_{j-1}, 1 \leq p \leq l_{j}^{q}} d_{1}\left(Q_{j, p}^{q}\right)
$$

To cover $E \cap V$. In fact $E \cap V \cap P_{j, i} \neq \emptyset$ for all the $k_{j}^{2}$ parallelepipeds $P_{j, i}$.

Since

$$
k_{j} \geq\left(\min _{1 \leq q \leq N_{j-1}, 1 \leq p \leq l_{j}^{q}} d_{1}\left(Q_{j, p}^{q}\right)\right)^{-2 j+1}
$$

we have

$$
\left(k_{j}\right)^{2 j} \geq\left(h_{j}\right)^{1-2 j}
$$

Thus, $\operatorname{dim}(E \cap V)=2$ which completes the proof because the set $B$ can be chosen such that for all $V \in B$ the set $\left\{a \in V^{\perp}: V_{a} \in A_{3,2}\left(P_{1,1}\right)\right\}$ is open.

Remark 14. All results proved in this section can be extended to higher dimensions.

In this section, we have studied the relationship between the Minkowski dimension of a set and those of its slices and we have shown that for Suslin sets they are different and they take account of local properties of the concerned set.

\section{Application to medical imaging}

To validate these theoretical results on real images, we study, in the next section the variation of 3D fractal dimension of volume versus its projections or slices. We choose three classes of images. The first is a self-similar fractal object called "Cantor set". The second class consists of synthetic models generated by a random process. Finally, we consider trabecular bones obtained from CT scan images.

\subsection{Fractal structure of trabecular bone}

There is no universal and unique definition of a "fractal" but "we know one when we see one". However, we can characterize fractal curves [19]. For a given curve $\Gamma$ of parameterization continuous map (Figure 2) $\gamma:[a, b] \rightarrow \mathbb{R}$, if the ratio $\frac{T(t, \tau)}{\tau}$ goes to infinity when $\tau$ is so small we say that $\Gamma$ is fractal where $T$ is defined as follows.

For $t \in[a, b]$,

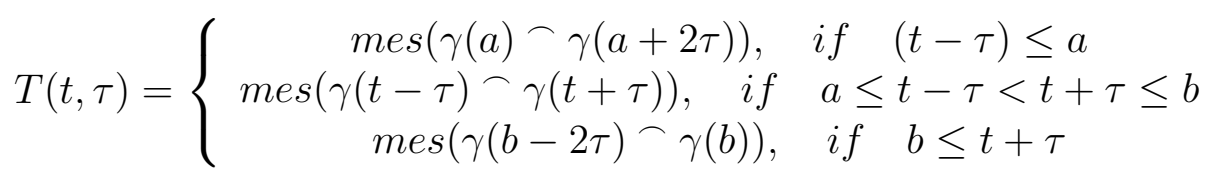




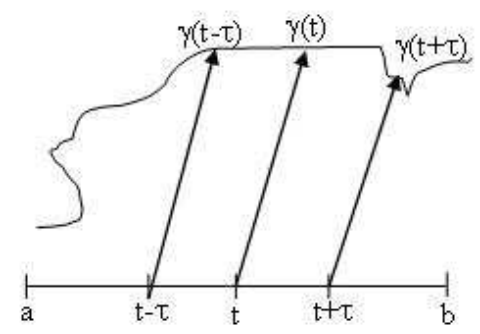

Figure 2: Curve $\Gamma$

The fractal arc $\gamma(t-\tau)^{\frown} \gamma(t+\tau)$ constructed around the point $\gamma(t)$ is the image of the temporal window $[t-\tau, t+\tau]$ by the parameterization $\gamma$.

If we take a profile $Z$ of our volume a calculus of the ratio $\frac{T(t, \tau)}{\tau}$ has shown us that it exploses as $\tau$ is so small. So, we can deduce that the image in grey level of a bone volume has rather a fractal structure.

This argues for choosing to study the fractal analysis of the bone texture and as an application to the results given in section 3 and 4 we show that in general there is no relationship between the 3D fractal dimension of a trabecular bone volume reconstructed from tomographic images obtained under routine clinical conditions and the $2 \mathbf{D}$ fractal dimension of its projections and sections under several directions. For this aim, we have modelized the trabecular bone by self-similar and selfaffine sets.

\subsection{CT Imaging Acquisition}

CT scan wrist images were obtained in 12 patients. Twenty slices (with $0.5 \mathrm{~mm}$ slice thickness and $0.137 \times 0.137 \mathrm{~mm}$ as pixel size) are obtained for each patient (Figure 3 ). The reconstructed
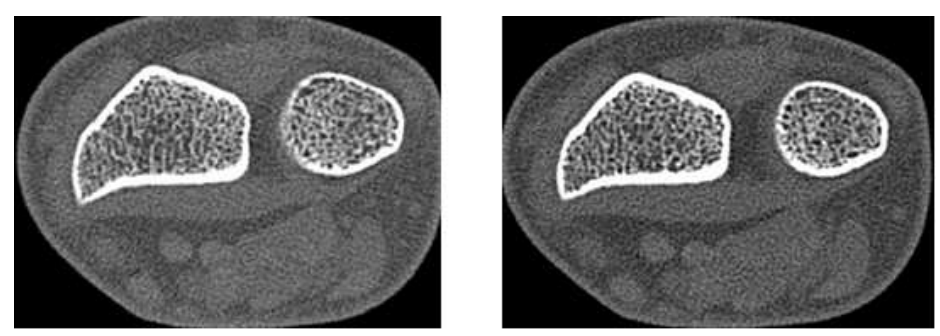

Figure 3: Two slices of CT scans wrist images 
bone volume by piling up native slices, has anisotropic voxels (size of pixels is different to slice thickness). To overcome this bias, we construct a bone volume, with cubic voxels, by superposing slices characterized by a thickness equal to their pixel size. These new slices are interpolated from native slices. For each of the 12 patients, a Volume Of Interest (VOI) (Figure 4), with $0.137 \times 0.137 \times 0.137 \mathrm{~mm}$ as voxel size, was extracted strictly covering the trabecular area.

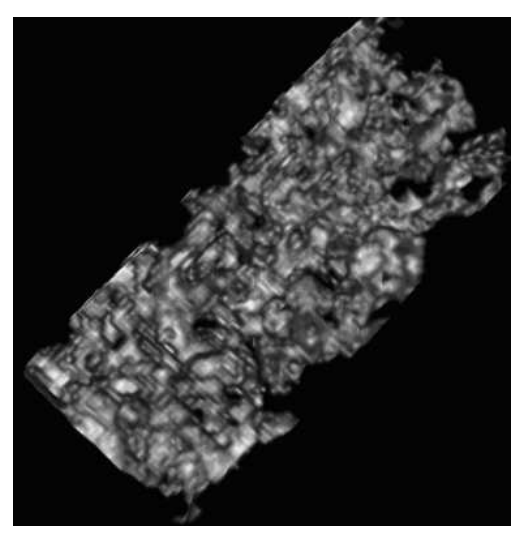

Figure 4: Volume Of Interest of trabecular bone

To compute the projection of these volumes, we use an orthogonal projection onto a specific plane. The projection of bone volumes generates grey level images.

\subsection{Modelization}

- The $K_{\frac{1}{3}}$ and $K_{\frac{1}{4}}$ Cantor sets

We choose as self-similar sets the $K_{\frac{1}{3}}$ and $K_{\frac{1}{4}}$ Cantor sets (Figure 5) as defined in section 3 (see Cantor set $K_{d}, 0<d<\frac{1}{2}$ ). Their respective Minkowski dimensions are equal to $\frac{\log 8}{\log 3}$ and $\frac{\log 8}{\log 4}$.

We consider the projection of these sets under several planes having an angle $\theta \in[0, \pi]$ with the Cartesian coordinate plane xoy.

If we take planes having an angle $\theta \equiv \frac{\pi}{3} \bmod (\pi)$ with $x o y$, the projected sets of $K_{\frac{1}{3}}$ have a Minkowski dimension equal to $\frac{\log 12}{\log 6}$. But the projection of $K_{\frac{1}{3}}$ onto the Cartesian coordinate planes has a Minkowski dimension equal to $\frac{\log 4}{\log 3}$. And if we consider the projection of $K_{\frac{1}{3}}$ onto a plane having an angle $\theta \equiv \frac{\pi}{4} \bmod (\pi)$ with $x o y$ the Minkowski dimension is $\frac{\log 6}{\log 3}$.

Similar calculations done with the $K_{\frac{1}{4}}$ Cantor set lead to the following results. The projected sets under Cartesian coordinate planes have Minkowski dimension equal to 1. For a plane having an angle $\theta \equiv \frac{\pi}{3} \bmod (\pi)$ with $x o y$ the projected sets have a Minkowski dimension equal to $\frac{\log 12}{\log 8}$ and for a plane having an angle $\theta \equiv \frac{\pi}{4} \bmod (\pi)$ the Minkowski dimension of the projections of $K_{\frac{1}{4}}$ is equal to $\frac{\log 8}{\log 4}$. 
As mentioned in section 3 there is no relationship between Minkowski dimension of a set and those of their projections and we remark, for the particular sets considered, that the dimensions of the projections differ from a given set of planes to an other.

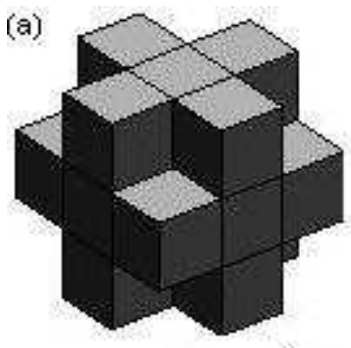

(b)

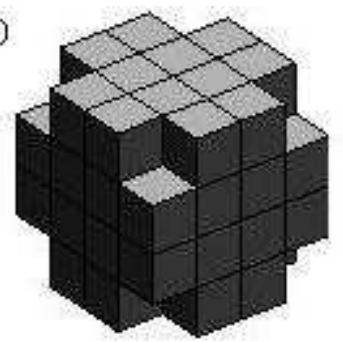

Figure 5: (a) Cantor $K_{\frac{1}{3}}$ and (b) Cantor $K_{\frac{1}{4}}$

\section{- Self-affine models}

As a self-affine model, we choose the Sierpinski sponges and probability measures supported on these sets (simulating the grey level in the bone images). These sets are generated as follows. Let $1 \leq n_{1} \leq n_{2} \leq n_{3}$ be integers. For each $l=1,2,3$ the unit cube $[0,1]^{3}$ is partitioned into $n_{1} n_{2} n_{3}$ congruent boxes with sidelengths $\frac{1}{n_{1}}, \frac{1}{n_{2}}, \frac{1}{n_{3}}$. Let $\mathcal{B}$ be a subcollection of these boxes and let $p=\left(p_{B}\right)_{b \in \mathcal{B}}$ be a probability vector indexed by $\mathcal{B}$. Erase all the boxes not contained in $\mathcal{B}$ and divide a unit mass between the remaining boxes in the ratio determined by the probability vector $p$. Then each of the remaining boxes $B$ is partioned into $n_{1} n_{2} n_{3}$ congruent subboxes of $B$ with side-lengths $\frac{1}{n_{1}^{2}}, \frac{1}{n_{2}^{2}}, \frac{1}{n_{3}^{2}}$ again keeping only those which correspond to $\mathcal{B}$ and divide the mass of $B$ between the remaining subboxes of $B$ in the ratio determined by the probability vector $p$. Continuing this process infinitely, a compact set $K$ and a probability measure supported on $K$ are obtained (Figure 6). The set $K$ is called a self-affine Sierpinski sponge.

We also consider the projections and sections of these sets under several directions. These models are generated under Matlab ${ }^{T M}$ software and the probability vector $p$ is chosen randomly with probability $p r$ for the partition of "white level".

\subsection{Methods of calculation of the $2 \mathrm{D}$ and $3 \mathrm{D}$ fractals dimensions of the mod- els}

\subsubsection{Theoretical method of calculating the fractal dimension}

Numerical studies have been carried out on the calculation of Minkowski dimensions for some self-affine sets (e.g. Sierpinski sponges). In 1984, C. McMullen gave a formula to calculate the fractal dimensions $D_{t h}(2 D)$ of these models in two dimensions [16]. 
(a)

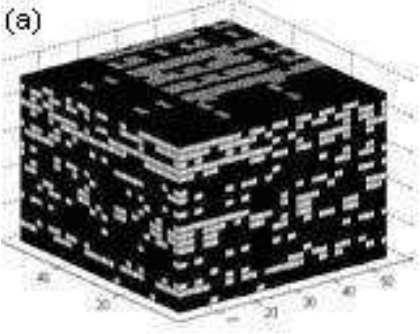

(b)

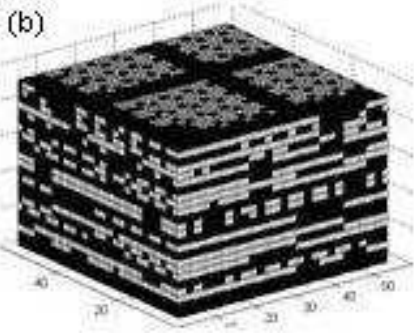

(c)

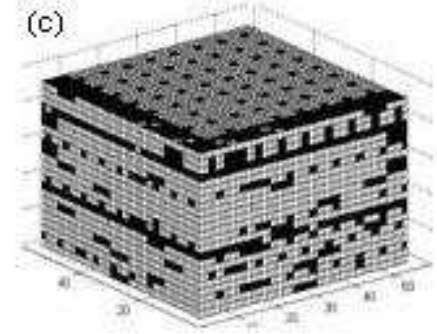

Figure 6: Models with (a) pr=0.3, (b) pr=0.7 and (c) pr=0.9.

Let $n_{1}$ and $n_{2}$ designate the dimensions of the image and the grey level of pixel $(i, j)$ is denoted by $N G(i, j)$. Then if $n_{1}<n_{2}$, one has

$$
D_{t h}(2 \mathbf{D})=\frac{1}{\log n_{1}} \log \left(\frac{r}{s}\right)+\frac{1}{\log n_{2}} \log s
$$

Where $r=\operatorname{card}\{(i, j): N G(i, j) \neq 255\}, s=\operatorname{card}\{j: \exists i ; N G(i, j) \neq 255\}$, and 255 is the grey level of the white intensity in the image. This formula has been generalized by Olsen [17] to higher dimensional space.

The theoretical 3D Minkowski dimension of a self-affine model is calculated using the following expression:

$$
D_{t h}(3 \mathbf{D})=\left(\frac{1}{\log n_{1}}-\frac{1}{\log n_{2}}\right) \log u+\left(\frac{1}{\log n_{2}}-\frac{1}{\log n_{3}}\right) \log v+\frac{\log w}{\log n_{3}}
$$

Where $n_{1}, n_{2}$, and $n_{3}$ are the dimensions of the volume with $n_{1}<n_{2}<n_{3}$ and

$u=\operatorname{card}\{j: N G(i, j, k) \neq 255\}$ Provided that for every $(i, k)$ a retained value of $j$ is counted only once.

$v=\operatorname{card}\{(i, j): N G(i, j, k) \neq 255\}$ Provided that for every $k$ a retained value of $(i, j)$ is counted only once.

$$
w=\operatorname{card}\{(i, j, k): N G(i, j, k) \neq 255\} .
$$

\subsubsection{Minkowski-Bouligand dimension}

The concept of Minkowski-Bouligand dimension, was introduced by G.Bouligand [4].

Let $E$ be a bounded set in the 3-dimensional Euclidean space, if we denote the volume of a set $F$ by $V(F)$, the Minkowski-Bouligand dimension is given by:

$$
D_{m b}(3 \mathbf{D})=\lim _{\varepsilon \rightarrow 0}\left(3-\frac{\log V(A(\varepsilon))}{\log \varepsilon}\right)
$$


$A(\varepsilon)$ is also called the Minkowski sausage of $E$.

Let us give a numerical method to calculate $V(A(\varepsilon))$. In the case of a $2 \mathbf{D}$ grey level image noted by $M$, we cover this image by the application of successive series of erosions (with a circle as structuring element). For every erosion we measure the surface area $S(\varepsilon)$ ( $\varepsilon$ is the radius of the circle) of the eroded image $M_{\varepsilon}$. One has,

$$
S(\varepsilon)=\frac{V(\varepsilon)}{\varepsilon^{2}}
$$

$V(\varepsilon)$ is given by

$$
V(\varepsilon)=\sum_{x=1}^{N} \sum_{y=1}^{N}\left(M(x, y)-M_{\varepsilon}(x, y)\right)
$$

Where $N$ is the size of $M$. The slope of the regression line $(\log \varepsilon, \log (S(\varepsilon)))$ corresponds to $D_{m b}(2 \mathbf{D})$. In the case of a $3 \mathbf{D}$ structure, the same process is applied and we measure the surface area $S(\varepsilon)$ of the eroded image such as

$$
S(\varepsilon)=\frac{V(\varepsilon)}{\varepsilon^{3}}
$$

$V(\varepsilon)$ is expressed by

$$
V(\varepsilon)=\sum_{x=1}^{N} \sum_{y=1}^{N} \sum_{z=1}^{N}\left(M(x, y, z)-M_{\varepsilon}(x, y, z)\right)
$$

The slope of the plot $(\log \varepsilon, \log (S(\varepsilon)))$ corresponds to $D_{m b}(3 \mathbf{D})$.

All calculations here under presented were made on grey scale images(slices or projections)without any binarisation by thresholding.

\subsection{Relationship between the 3D fractal dimensions of a volume and their projections}

\subsubsection{Application to models}

Both 2D and 3D Minkowski dimensions are estimated on 18 self-affine models obtained for different probability values $p r$ and on their projections using 5.2 and 5.3. We search if the 3D theoretical Minkowski dimension and 2D ones of their projections are linked and if we can deduce the theoretical Minkowski dimension of a 3D structure from those of its projections. We denote by $D_{t h, p}(2 \mathbf{D})$ the $2 \mathbf{D}$ theoretical Minkowski dimension of the projection of a set. We represent the variation of $D_{t h}(3 \mathbf{D})$ versus $D_{t h, p}(2 \mathbf{D})$ for all models and for each projection. Figure 7 shows an example of this plot when considering the projection onto the plane xoz. We remark that there is no direct relation between the two parameters $D_{t h}(3 \mathbf{D})$ of volume and $D_{t h, p}(2 \mathbf{D})$ of those projections. To confirm this, we describe plots obtained for each projection with a polynomial equation. 


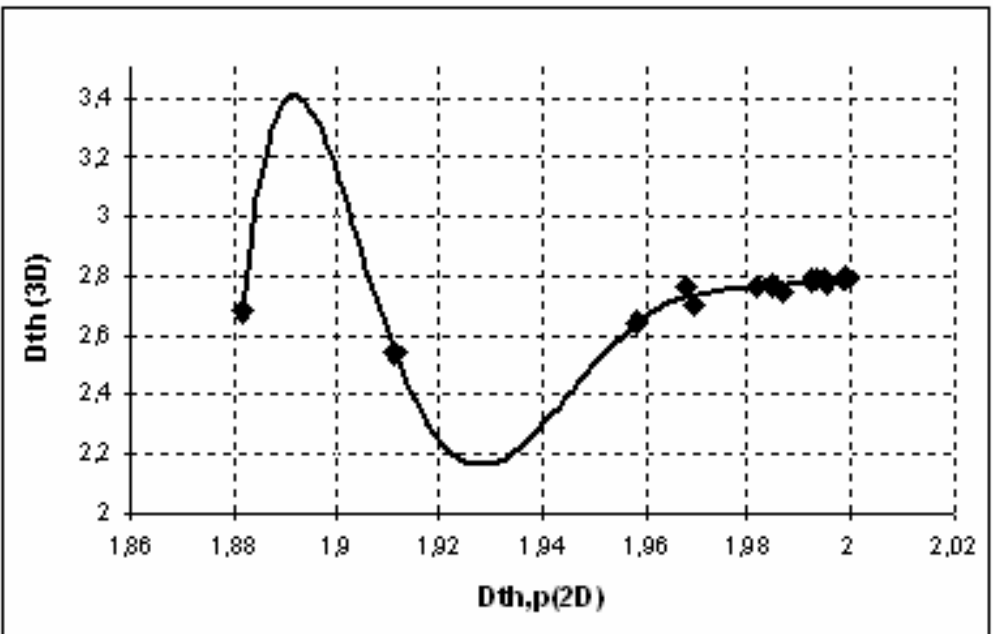

Figure 7: $D_{t h}(3 \mathbf{D})$ of models versus $D_{t h, p}(2 \mathbf{D})$ of its projections onto xoz.

Table 1: Relation between $D_{t h}(3 \mathbf{D})$ and $D_{t h, p}(2 \mathbf{D})$ of models

\begin{tabular}{|c|c|c|c|c|}
\hline & Proj. onto $x o z$ & Proj. onto yoz & Proj. onto xoy & Proj. onto $x=y$ \\
\hline Polynomial degree & 6 & 6 & 3 & 10 \\
\hline Correlation coefficient & 0.969 & 0.918 & 0.205 & 0.993 \\
\hline
\end{tabular}

We present in table 1 the degree of the polynom obtained for each projection and the coefficient of correlation. We notice that the choice of regression curves as polynomial ones is not arbitrary. We have tested other regression curves (linear, exponential) and we have chosen, for each representation, the interpolation with higher correlation coefficient, closest to 1 . These results confirm the theoretical results, that there is no monotonous relation between $3 \mathbf{D}$ fractal dimension of a volume and its $2 \mathbf{D}$ projections.

\subsubsection{Application to CT scan images}

In the light of a new theoretical result shown in this paper, and confirmed on models, we experimentally examine this result. We calculate fractal dimension by using the Minkowski method for bone volume and the same method is used for its projections onto each plane. We denote by $D_{m b, p}(2 \mathbf{D})$ the 2D Minkowski-Bouligand dimension of the projection of a set. Figure 8 shows a representation of $D_{m b}(3 \mathbf{D})$ onto xoz plane versus $D_{m b, p}(2 \mathbf{D})$ for all bone volumes. 
Table 2: Relation between $D_{m b}(3 \mathbf{D})$ and $D_{m b, p}(2 \mathbf{D})$ of CT scan images

\begin{tabular}{|c|c|c|c|c|}
\hline & Proj. onto xoz & Proj. onto yoz & Proj. onto xoy & Proj. onto $x=y$ \\
\hline Polynomial degree & 6 & 6 & 6 & 6 \\
\hline Correlation coefficient & 0.876 & 0.926 & 0.757 & 0.962 \\
\hline
\end{tabular}

We calculate a polynom describing the variation of $D_{m b}(3 \mathbf{D})$ onto the plane xoy versus $D_{m b, p}(2 \mathbf{D})$ of its projections (See Table 2).

We obtain polynoms for each projection with the same degree of polynoms but with different coefficients. Then, the relation between $D_{m b}(3 \mathbf{D})$ of volume and $D_{m b, p}(2 \mathbf{D})$ of its projections is not constant and differs for all patients.

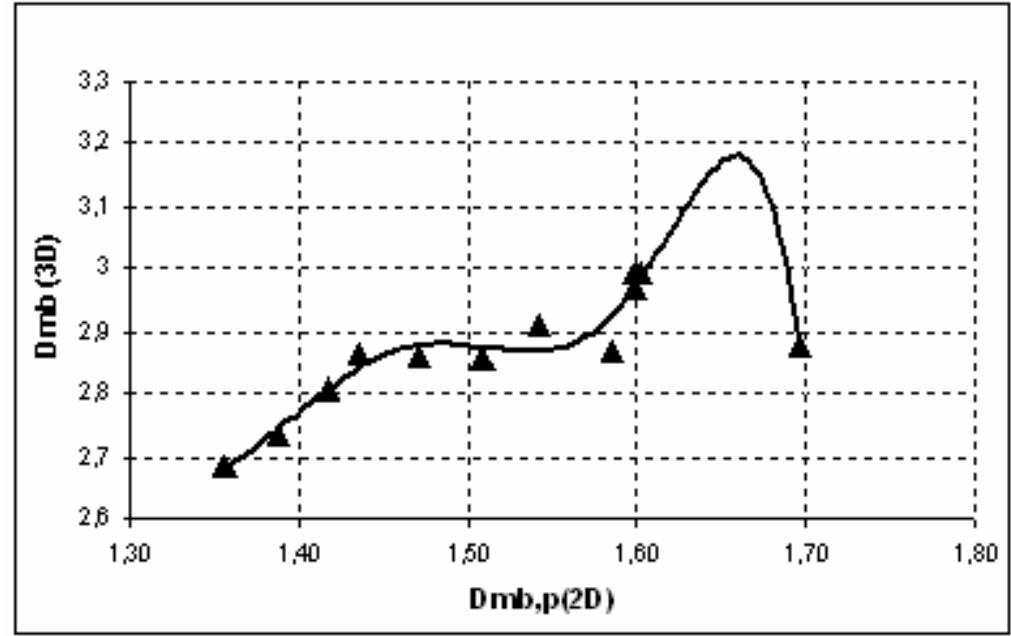

Figure 8: $D_{m b}(3 \mathbf{D})$ of bone volume versus $D_{m b, p}(2 \mathbf{D})$ of its projections onto xoz.

From a value of $D_{m b, p}(2 \mathbf{D})$ of any plane (case of $2 \mathbf{D}$ image obtained by radiographic imaging) we cannot deduce a $D_{m b}(3 \mathrm{D})$ of a volume and describe the whole of trabecular bone.

\subsection{Relationship between the 3D fractal dimensions of a volume and their slices}

As mentioned before, the aim of this work is to evaluate the pertinence of a fractal analysis of a bone volume computed from tomographic images. Before this, it is important to verify if we 
obtain the same fractal dimension for the bone volume and for its slices or if we can deduce, for all patients, a relationship between their fractal dimensions 3D and 2D.

\subsubsection{Application to models}

We denote by $D_{t h, s}(2 \mathbf{D})$ the $2 \mathbf{D}$ theoretical Minkowski dimension of a slice of a set. For each model, we calculate the $D_{t h, s}(2 \mathbf{D})$ of each slice obtained from the volume along z-axis. We represent $D_{t h}(3 \mathbf{D})$ versus $D_{t h, s}(2 \mathbf{D})$, for some models and for all their slices. We note that, for the clarity of the figure, we present in Figure 9 results obtained for some models but it was verified for all models.

First, we remark that for a value of $D_{t h, s}(2 \mathbf{D})$ of any slice we deduce more than one value of $D_{t h}(3 \mathbf{D})$ in the ordinate axis. For example, for $D_{t h, s}(2 \mathbf{D})=1.84$ three values of $D_{t h}(3 \mathbf{D})(2.64$, 2.71 and 2.75) are obtained from points of figure 9. Second, the relation between the two values $D_{t h}(3 \mathbf{D})$ of volume and $D_{t h, s}(2 \mathbf{D})$ of its slices depends on the position of slices. Then, we can't deduce $D_{t h}(3 \mathbf{D})$ of a volume from the $D_{t h, s}(2 \mathbf{D})$ of the slice.

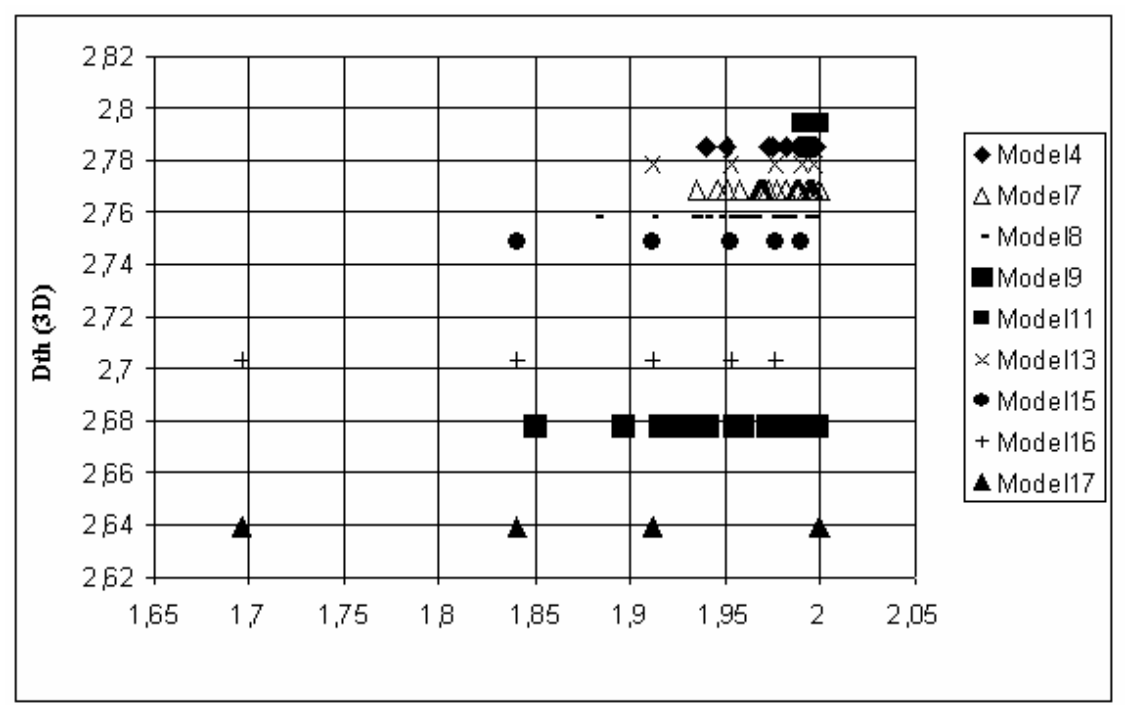

Figure 9: $D_{t h}(3 \mathbf{D})$ of models versus $D_{t h, s}(2 \mathbf{D})$ of its slices.

\subsubsection{Application to CT scan images}

Let us verify this result for real images, CT scan images of 12 patients. Figure 10 presents fractal dimension of 3D volume bone versus fractal dimension of $2 \mathbf{D}$ of its parallel slices obtained along z-axis. We note that, for the clarity of figure, we have presented results obtained for only 8 patients but all of the other patients results have been verified. 
We can observe in Figure 10 the dispersion of 2D fractal dimension of slices for each patient. We denote by $D_{m b, s}(2 \mathrm{D})$ the $2 \mathbf{D}$ Minkowski dimension of a slice of a set. Different values of $D_{m b}(3 \mathbf{D})$ of bone volume are obtained from one value of $D_{m b, s}(2 \mathbf{D})$. So, we cannot deduce any relation between these two dimensions $\left(D_{m b}(3 \mathbf{D})\right.$ of volume and $D_{m b, s}(2 \mathbf{D})$ of its slices).

We conclude that in clinical application and in the case of tomographic imaging (CT scan and MRI) it is impossible to characterize a bone trabecular volume from a unique value of fractal dimension of slices of bone volume.

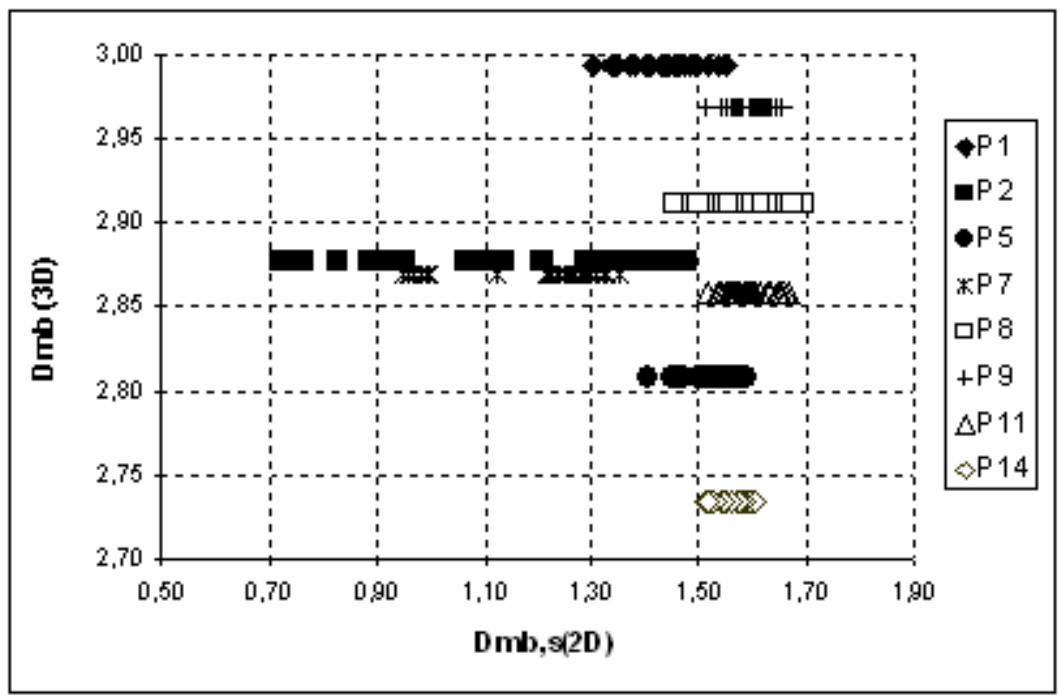

Figure 10: $D_{m b}(3 \mathbf{D})$ of bone volume versus $D_{m b, s}(2 \mathbf{D})$ of its CT slices.

\section{Conclusion}

The current methods of medical imaging make it possible to better understand with more accuracy the reality of the structures of organs. The $3 \mathbf{D}$ reconstruction obtained from $2 \mathbf{D}$ images poses many methodological questions and in particular concerning quantization.

The fractal approach of the trabecular bone is attractive, easy to implement and allows a measurement of the complex variation of bone micro architecture. In this paper, we have shown that there is no possibility, in the general case, to find an exact link between the 3D fractal dimension of sets and the 2D fractal dimension of its projections or slices. This gives a generalization of the result of [8] concerning the relationship between the 3D self-similarity of an isotropic continuous 
fractional brownian motion process and the 2D self-similarity of its projections only onto the three orthogonal directions. As proved in sections 3 and 4, the Hausdorff measure and Minkowski contents of projected sets and the slices have unstable behaviour and depend on the local properties of the considered set. Moreover, the relation between the fractal dimension of a volume and that of its projections changes with the direction of projection. Those results are also proved for synthetic models and for CT scan images.

In clinical applications, this result shows that a radiographic projection of trabecular bone is not sufficient and cannot describe the totality of bone micro architecture.

In the same way, the fractal dimension of a slice of bone (CT scan or MRI imaging) is not an appropriate parameter for characterizing bone volume. Results proved in this paper justify the necessity of analyzing trabecular volume and not only one radiographic projection or some slices.

A projection or a slice gives information about just that part and not about the whole volume. In the case of osteoporosis, degradation of bone micro architecture is not restricted to one direction. So, 2D images obtained by radiographic or tomographic imaging are limited in the description of the whole variation in trabecular bone. Also, theoretical and practical results proved in this paper demonstrate that a quantification on a simple plane (projection or slice) by fractal dimension is not sufficient for characterizing the volume of trabecular bone.

We are currently working on 3D fractal and multifractal methods to better quantify bone micro architecture.

\section{Appendix}

In this section we give respectively the proofs of lemma 6 , lemma 8 and lemma 13.

Proof of lemma 6. The assumption (1) follows from $\mathcal{L}^{2}\left(K_{i}\right)=\frac{1}{m^{2}} \mathcal{L}^{2}(K)$.

Let us prove the assumption $(2)$. Recall that $A_{\varepsilon}(E)$ denotes the neighbourhood of radius $\varepsilon$ of a set $E$. Suppose that $K_{l}$ are pairwise disjoint. Since they are compact, the distance between any given two of them is positive. Then, there exists $\varepsilon>0$ such that

$$
A_{\varepsilon}\left(K_{i}\right) \cap A_{\varepsilon}\left(K_{j}\right)=\emptyset \text { for } i \neq j
$$

Thus we have

$$
\mathcal{L}^{2}\left(A_{\varepsilon}(K)\right)=\mathcal{L}^{2}\left(\bigcup_{l=1}^{m^{2}} A_{\varepsilon}\left(K_{l}\right)\right)=\sum_{l=1}^{m^{2}} \mathcal{L}^{2}\left(A_{\varepsilon}\left(K_{l}\right)\right)
$$

On the other hand,

$$
\mathcal{L}^{2}\left(A_{\varepsilon}\left(K_{l}\right)\right)=\mathcal{L}^{2}\left(A_{\varepsilon}\left(\frac{1}{m} K+d_{l}\right)\right)=\mathcal{L}^{2}\left(A_{\varepsilon}\left(\frac{1}{m} K\right)\right)=\frac{1}{m^{2}} \mathcal{L}^{2}\left(A_{m \varepsilon}(K)\right)
$$

since $m A_{\varepsilon}\left(m^{-1} K\right)=A_{m \varepsilon}(K)$.

So, $\mathcal{L}^{2}\left(A_{\varepsilon}(K)\right)=\mathcal{L}^{2}\left(A_{m \varepsilon}(K)\right)$ which contradicts the fact that $A_{\varepsilon}(K)$ is a proper subset of $A_{m \varepsilon}(K)$. 
Proof of lemma 8. Suppose that $\mathcal{L}^{2}(K)>0$ then there exists a square $C$ such that $\mathcal{L}^{2}(K \cap C)>$ $0.9 \mathcal{L}^{2}(C)$ (this follows immediately from Lebesgue's theorem on points of density). Let $\varepsilon$ smaller enough and $K_{u}$ and $K_{v}, u, v \in I^{l}, u \neq v$ which are $\varepsilon$-relatively close. Then the quadrilateral $m^{-l} C+d_{u}$ and $m^{-l} C+d_{v}$ have a large overlap since $\varepsilon$ is small. But $K_{u}$ and $K_{v}$ fill 90 percent of each of them which is impossible since $\mathcal{L}^{2}\left(K_{u} \cap K_{V}\right)=0$ and the lemma is proved.

Proof of lemma 13. We denote by $\gamma_{w_{3}, 1}$ the invariant measure on the grassmannian manifold $G\left(w_{3}, 1\right)$ of all lines of $w_{3}$. Due to lemma 12 and by identifying $w_{3}$ with $\mathbb{R}^{2}$ and defining $A_{w_{3}, 1}(\tilde{P})$ and $A_{w_{3}, 1}^{\delta}(\tilde{P})$ in the obvious way for hyperregular parallelograms $\tilde{P} \subset[0,1]^{2}$ in $w_{3}$ and for $\delta>0$, we find disjoint compact sets $\tilde{A}, \tilde{B} \subset G\left(w_{3}, 1\right)$ with $\gamma_{w_{3}, 1}(\tilde{A})>0$ and $\gamma_{w_{3}, 1}(\tilde{B})>0$ such that for all hyperregular parallelograms $\tilde{P} \subset[0,1]^{2}$ and for all $\varepsilon>0$, there exists a finite family $\tilde{P}_{\varepsilon}$ of hyperregular subparallelograms of $\tilde{P}$ such that

$$
\mathcal{H}^{1}\left(p_{V^{\perp}, w_{3}}\left(\cup \tilde{P}_{\varepsilon}\right)\right) \leq \varepsilon \quad \text { for } \quad \text { all } \quad V \in \tilde{A}
$$

Here $p_{V^{\perp}, w_{3}}: w_{3} \rightarrow V^{\perp, w_{3}}$ is the orthogonal projection onto the orthogonal complement $V^{\perp, w_{3}} \in G\left(w_{3}, 1\right)$ of $V$. Moreover, there is $\delta>0$ such that if $V \in A_{w_{3}, 1}(\tilde{P}) \cap A_{w_{3}, 1}\left([0,1]^{2}\right)$ is parallel to some line belonging to $\tilde{B}$ then there exists $\tilde{Q} \in \tilde{P}_{\varepsilon}$ such that

$$
V \in A_{w_{3,1}}(\tilde{Q})
$$

Define $A=\left\{V \in G(3,2): p_{w_{3}}(V) \in \tilde{A}\right\}$ and $B=\left\{V \in G(3,2): p_{w_{3}}(V) \in \tilde{B}\right\}$.

Since

$\gamma_{3,2}(A)=c \mathcal{H}^{3} \times \mathcal{H}^{3}\left(\left\{\left(y_{1}, y_{2}\right) \in\left(\mathbb{R}^{3}\right)^{2}:\left|y_{i}\right| \leq 1 \quad\right.\right.$ for $\quad i=1,2 \quad$ and $\left.\left.\operatorname{vect}\left(y_{1}, y_{2}\right) \in A\right\}\right)$

where vect $\left(y_{1}, y_{2}\right)$ is the plane spanned by $\left(y_{1}, y_{2}\right)$, then $\gamma_{3,2}(A)>0$ and similarly $\gamma_{3,2}(B)>0$. Indeed, $\gamma_{w_{3}, 1}(\tilde{A})>0$ and $\gamma_{w_{3}, 1}(\tilde{B})>0$. Let $P \subset[0,1]^{3}$ be a hyperregular parallelepiped and $\varepsilon>0$, due to the fact that $\tilde{P}=P \cap w_{3}$ is a hyperregular parallelograms in $w_{3}$ then by identifying $w_{3}$ with $\mathbb{R}^{2}$, there exists a finite family $\left\{\tilde{P}_{\varepsilon}^{1}, \ldots, \tilde{P}_{\varepsilon}^{k}\right\}$ of hyperregular subparallelograms of $\tilde{P}$ satisfying (7.4) and (7.5). The family $P_{\varepsilon}=\left\{[0,1] \times \tilde{P}_{\varepsilon}^{1}, \ldots,[0,1] \times \tilde{P}_{\varepsilon}^{k}\right\}$ is a finite family of hyperregular subparallelepipeds of $P$. Consider $V \in A$, note that $p_{V^{\perp}}\left(\cup \tilde{P}_{\varepsilon}\right)=p_{V^{\perp}} p_{\left(V \cap w_{3}\right)^{\perp}}\left(\cup \tilde{P}_{\varepsilon}\right)=$ $p_{V^{\perp}} p_{\left(V \cap w_{3}\right)}^{\perp, w_{3}}\left(\cup \tilde{P}_{\varepsilon}\right)$.

So,

$$
\mathcal{H}^{1}\left(p_{V \perp}\left(\cup \tilde{P}_{\varepsilon}\right)\right) \leq \varepsilon
$$

And the first assertion holds.

Finally, if $V \in A_{3,2}(P) \cap A_{3,2}\left([0,1]^{3}\right)$ is parallel to some plane belonging to $B$, then for $i=1,2$ we have $p_{w_{3}}\left(V \cap P_{i}^{j}\right)=p_{w_{3}}(V) \cap \tilde{P}_{i}^{j}$ for $j=1,2$. Since $p_{w_{3}}(V) \in A_{w_{3}, 1}(\tilde{P}) \cap A_{w_{3}, 1}\left([0,1]^{2}\right)$ is parallel to some plane belonging to $\tilde{B}$, we obtain by (7.5) that $p_{w_{3}}(V) \in A_{w_{3}, 1}\left(\tilde{P}_{\varepsilon}^{l}\right)$ for some $1 \leq l \leq k$, giving $V \in A_{3,2}\left([0,1] \times \tilde{P}_{\varepsilon}^{l}\right)$. 


\section{References}

[1] C. Bandt, S. Graf. Self similar sets VII. A characterization of self-similar fractals with positive hausdorff measure. Proc Amer.Math.Soc., 114 (1992), No. 4, 995-1001.

[2] L. Carleson. Selected problems on exceptionel sets. Van Nostrand, New York, 1968.

[3] D. Chappard, E. Legrand, B. Haettich, G. Chalès, B. Auvinet, J.P. Eschard, J.P. Hamelin, M.F. Baslé, M. Audran. Fractal dimension of trabecular bone: Comparison of three histomorphometric computed techniques for measuring the architectural two-dimensional complexity. Journal of Pathology, 195 (2001), 515-521.

[4] K.J. Falconer. Fractal geometry: Mathematical foundations and applications. John Willey \& Sons, New York, 1990.

[5] K.J. Falconer, J.D. Howroyd. Projection theorems for box and packing dimensions. Math.Proc.Cambrige philas.Soc., 119 (1996), 287-295.

[6] S. Herlidon, R. Grebe, F. Grados, N. Leuyer, P. Fardellone, M. E. Meyer. Influence of age and osteoporosis on calcaneus trabecular bone structure: A preliminary in vivo MRI study by quantitative texture analysis. Magnetic Resonance Imaging, 22 (2004), 237-243.

[7] J. Huntchinson. Fractals and self-similarity. Indiana Univ.math.J., 30 (1981), 713-747.

[8] R. Jennane, R. Harba, G. Lemineur, S. Bretteil, A. Estrade, C. L. Benhamou. Estimation of the $3 D$ self-similarity parameter of trabecular bone from its $2 D$ projection. Medical Image Analysis, 11 (2007), 91-98.

[9] R. Jennane, W. J. Ohley, S. Majumdar, G. Lemineur.Fractal analysis of bone X-ray Tomographic microscopy projections. IEEE Trans. Medical Imaging, 20 (2001), No. 5, 443-449.

[10] E. Lespessailles, C. Chappard, N. Bonnet, C. L. Benhamou. Imaging techniques for evaluating bone micro architecture. Joint Bone Spine, 73 (2006), 254-261.

[11] S. Majumdar, J. Lin, T. Link, J. Millard, P. Augat, X. Ouyang, D. Newitt, R. Gould, K. Mothari, H. Genant. Fractal analysis of radiographs: Assessment of trabecular bone structure and prediction of elastic modulus and strength. Med.Phys., 26 (1999), 1330-1340.

[12] J. M. Marstrand. Some fundamental geometrical properties of plane sets of fractional dimensions. Proc.London.Math-Soc, 3 (1954), No. 4, 257-302.

[13] P. Mattila. Hausdorff dimension, orthogonal projections and intersections with planes. Annales Acad.Sci.ten.A, 1 (1975), 227-244.

[14] P. Mattila. Smooth maps, null sets for integral geometric measure and analytic capacity. Ann. of Math., 123 (1986), 303-309. 
[15] P. Mattila. Geometry of sets and measures in Euclidean spaces. Cambridge University Press, Cambridge, 1995.

[16] C. Mc Mullen. The Hausdorff dimension of general Sierpinski carpets. Nagoya Math.J., 96 (1984), 1-9.

[17] L. Olsen. Self-Affine multifractal sierpinski sponges in $\mathbb{R}^{d}$. Pacific journal of mathematics, 183 (1998), No. 1, 143-199.

[18] L.Pothuaud, C. L.Benhamou, P.Porion, E. Lespessailles, R. Harba, P. Levitz. Fractal dimension of trabecular bone projection texture is related to three-dimensional micro architecture. J Bone Miner Res, 15 (2000), 691-699.

[19] C. Tricot. Courbes et dimensions fractales. Springer-Verlag, Heidelberg, 1999.

[20] A. Zaia, R. Eleonori, P. Maponi, R. Rossi,R. Murri. MR Imaging and osteoporosis: Fractal lacunarity analysis of trabecular bone. IEEE Transactions On Information Technology in Biomedecine, 10 (2006), No.3, 484-489. 